Revue d'histoire de l'Amérique française

REVUE D.HISTOIRE DE L'AMÉRIQUE FRANÇAISE

\title{
La contribution de Pierre Bouguer à la marine
}

\section{Roland Lamontagne}

Volume 17, numéro 1, juin 1963

URI : https://id.erudit.org/iderudit/302255ar

DOI : https://doi.org/10.7202/302255ar

Aller au sommaire du numéro

Éditeur(s)

Institut d'histoire de l'Amérique française

ISSN

0035-2357 (imprimé)

1492-1383 (numérique)

Découvrir la revue

Citer cet article

Lamontagne, R. (1963). La contribution de Pierre Bouguer à la marine. Revue d'histoire de l'Amérique française, 17(1), 87-92. https://doi.org/10.7202/302255ar d'utilisation que vous pouvez consulter en ligne.

https://apropos.erudit.org/fr/usagers/politique-dutilisation/ 


\section{LA CONTRIBUTION DE PIERRE BOUGUER A LA MARINE ${ }^{1}$}

Un dossier personnel de Pierre Bouguer existe sous la cote $\mathrm{C}^{7} 40$ aux Archives de France; les autres séries de la section ancienne ne semblent conserver aucun document émané de Pierre Bouguer; les inventaires des séries $\mathrm{AB}$ XIX et AP des archives privées ainsi que les fichiers du minutier central des notaires ont été examinés sans résultat positif.

Pierre Bouguer naquit au Croisic, le 10 février 1698, de Jean Bouguer et de Françoise Josseau. ${ }^{2}$ Après avoir servi dix ans à Brest sur les vaisseux, et avoir perdu une jambe au combat de Bantry, Jean Bouguer avait rempli pendant plus de vingt ans

${ }^{1}$ Nous exprimons notre vive gratitude à MM. Joël Audouy, Chef du Service des Archives et Bibliothèques du Ministère des Armées (Marine), Paris; François Dousset, Adjoint au Directeur général des Archives de France; Bernard Mahieu, Conservateur aux Archives de France; Henri Michel, Bibliothèque royale, Bruxelles; Murphy D. Smith, American Philosophical Society, Philadelphie, qui ont bien voulu nous fournir de précieuses indications pour ce travail. Nous remercions M. le Commandant Le Chuiton qui nous a adressé une photocopie annotée du ms. de la notice sur Pierre Bouguer que Prosper Levot, alors Conservateur de la Bibliothèque de la Marine à Brest, avait rédigée vers 1850 .

2 René Kerviler, Répertoire de bio-bibliographie bretonne (Rennes, 1891), V: 129-135; M. Prévost et Roman d'Amat, Dictionnaire de biographie française (Paris, 1954), VI: col. 1298-1299; Jean-Paul Grandjean de Fouchy, «Eloge de M. Bouguer», Histoire de l'Académie royale des Sciences (Paris, 1758), 127-136; Prosper Levot, Biographie bretonne (2 vol., Paris, 1852); M. l'abbé Rozier, Nouvelle table des articles contenus dans les volumes de l'Académie royale des Sciences de Paris depuis 1666 jusqu'en 1770, dans ceux des Arts et Métiers publiés par cette Académie et dans la Collection académique (4 vol., Paris, 1775-1776), IV: 41-45; Nouvelle biographie universelle (Paris, 1853), col. 909-910. Sur l'œuvre scientifique de Pierre Bouguer, voir Histoire générale des civilisations publiée sous la direction de Maurice Crouzet. V.-Le XVIII' siècle (Paris, 1953), 22-27; Histoire générale des sciences publiée sous la direction de René Taton. II. La science moderne (Paris, 1958), 484, 493, 502, 712; Histoire de la science publiée sous la direction de Maurice Daumas (Paris, 1957), 748-752, 911-912, 1651; Armand Letroye, Les académiciens français en Amérique méridionale au XVIII $e^{e}$ siècle (Léau, 1939) ; W. E. Knowles Middleton, édit., Pierre Bouguer's Optical Treatise on the Gradation of Light (Toronto, 1961). 
le poste de professeur d'hydrographie au Croisic. ${ }^{3}$ Il avait composé le Traité complet de la Navigation...4

Pierre Bouguer avait fait ses études au collège des Jésuites de Vannes. Le 27 juin 1714, il succédait à la place de professeur royal d'hydrographie, vacante par la mort de son père. ${ }^{5}$ Le $1^{\text {er }}$ décembre 1730, il sera nommé professeur d'hydrographie au Havre. ${ }^{6}$ Trois fois lauréat de l'Académie royale des Sciences, de 1727 à 1731, il recevra le titre d'associé géomètre de cette institution, le 5 septembre 1731 , et celui de pensionnaire astronome, le 26 février 1735.

Des pièces de ce dossier personnel mettent en lumière l'objet et la méthode de travail de Pierre Bouguer. Alors qu'il était professeur d'hydrographie au Croisic, Bouguer proposait de recueillir les observations relatives à l'histoire naturelle contenues dans les journaux de la marine et d'inciter les « Pilotes et Maîtres de Navire » à consigner les résultats d'ordre hydrographique. ${ }^{7}$

Des remarques sur la rigueur expérimentale, dans une lettre datée du 16 octobre $1730,{ }^{8}$ préfigurent les explications méthodologiques que Bouguer présente dans le texte de sa préface au Traité du Navire...: «qu'on ne se dissimule aucune circonstance, qu'on tente de résoudre les problèmes dans toute leur difficulté, et on verra un continuel accord entre la théorie et la pratique; le contraire impliqueroit contradiction . . . les matieres compliquées ne se perfectionnent que peu-à-peu et que

\footnotetext{
${ }^{3}$ Archives de France, Marine $\mathrm{C}^{7} 40$, fo 10.

${ }^{4} \mathrm{La}$ Bibliothèque Saint-Sulpice de Montréal possède une copie de la seconde édition de ce traité.

5 Archives de France, Marine C 70 , fo 1 .

6 Ibid., fo 14.

7 « Ces observations pourroient être dans la suite d'un grand secours pour les Marins; et, si elles ne répandroient pas de la lumiere sur la Physique, elles aprendroient au moins toujours des faits et serviroient à perfectionner l'Histoire naturelle. On trouveroit, peut être, encore d'autres avantages: car on pourroit charger les Pilotes de faire des remarques sur la situation et sur le gisement des terres qu'ils verroient, et ces remarques rassemblées en grand nombre, comparées les unes avec les autres, et jointes avec les determinations exactes que le Roy a fait faire des principaux Caps et des endroits les plus considerables, nous metroient bientôt en état de former des cartes hydrographiques plus exactes que celles que nous avons. $>$ Ibid., fo 4 .

8 Archives de France, Marine $\mathrm{C}^{7} 40, \mathrm{f}^{\circ} 12 \mathrm{v}$ et 13.
} 
par parties $\$^{9}$ La science expérimentale consiste à vérifier les déductions mathématiques par l'observation et l'expérience, en éliminant les théories contredites par les faits; elle ouvre la voie à de nouvelles découvertes. M. le $\mathrm{P}^{\mathrm{r}}$ Louis de Broglie précise ainsi la nature de la méthode expérimentale: «Partant d'idées ou de principes suggérés par l'observation ou par l'expérience, elle [la méthode expérimentale] en avait tiré à l'aide de déductions mathématiques la prévision de phénomènes nouveaux dont l'observation ou l'expérience, intervenant derechef, était venue contrôler la réalité. $\gg^{10}$

Dans une lettre de félicitations au nouveau ministre de la Marine, Antoine-Louis Rouillé qui succédait à Maurepas, Pierre Bouguer profite de cette occasion pour faire valoir les faits marquants de sa carrière, en date du 11 mai 1749:

Le Roy envoya en 1735 plusieurs Academiciens au Pérou: mais lorsqu'on fut à la veille de partir, plusieurs de ceux qui s'etoient offerts, ne purent satisfaire à l'engagement qu'ils avoient pris, et le voyage courroit risque de manquer. On pensa à moi: j'étois alors Hydrographe au Havre de grace; et trois prix de l'Academie des sciences que j'avois remportés successivement sur des matieres de Marine, m'avoient depuis quatre ou cinq ans procuré l'honneur d'entrer dans cette compagnie. Je montrai assez de bonne volonté pour faire mes préparatifs à la hate; et je quittai une vie assez tranquile, pour en embrasser une très pénible et extremement agitée, pendant neuf ans qu'a duré ce voyage. Vous sçavez, Monseigneur, qu'il s'agissoit d'observations utiles à l'astronomie et à la Physique et qui ne devroient pas l'être moins à la Navigation. Quelques mois après mon retour, M. de Maupertuis qui etoit préposé pour travailler à la perfection des instrumens et des pratiques des Pilotes, et généralement de tout ce qui dans la Marine a quelque raport aux Mathématiques, passa en Prusse, et laissa vacante la place qu'il occupoit. M. le comte de Maurepas me fit l'honneur de jetter les yeux sur moi pour la remplir, ce qui m'a

9 Pierre Bouguer, Traité du Navire, de sa construction et de ses mouvemens (Paris, 1746), xiii.

10 Savants et Découvertes (Paris, 1951), 32. 
déterminé à m'appliquer encore d'avantage à la Marine. J'ay publié depuis mon arrivée un assez gros volume sur la construction des vaisseaux et sur la théorie de leurs manoeuvres; et je me suis engagé d'en donner un autre sur le Pilotage. ${ }^{11}$

Comme on vient de le constater, Pierre Bouguer insiste sur sa contribution à la Marine; les possessions françaises ressortissaient à ce ministère. Selon Roland-Michel Barrin de La Galissonière, alors gouverneur intérimaire de la Nouvelle-France, l'ouvrage de Pierre Bouguer devait servir au chantier maritime de Québec.

On auroit été agreablement surpris en France d'y voir arriver le $\mathbf{S}^{\mathrm{t}}$ Laurent dont l'armement avoit eté contremandé, mais les ordres sont venus trop tard et il n'y avoit plus moyen de reculer. Mandé moy ce qu'on auroit dit de ce vaisseau, je m'y interesse a cause du constructeur ${ }^{12}$ qui est un tres bon sujet. Il a demandé le livre de M. Bouguer et je suis persuadé qu'il en fera un bon usage, car il n'est pas entêté comme la pluspart de ses confreres. ${ }^{13}$

La lettre d'où est tiré le fragment, que nous venons de lire, donne d'intéressantes précisions sur l'organisation matérielle du Canada. Aux yeux de La Galissonière, l'essor industriel de la colonie est lié à l'accroissement démographique. «Pour la potasse et pour les cendres de soude et de plantes marines équivalentes, ainsi que pour le savon et les verreries, je crois qu'il faut attendre que le pays soit plus peuplé; les atteliers des forges et celui de la construction [navale] qui sont d'une bien autre conséquence ne battent que d'une aile et tomberoient tout a fait si on n'envoioit pas continuellement du monde de france.»

Dès 1736, Henri-Louis Duhamel du Monceau était satisfait de la qualité des navires sortis des cales de Québec. ${ }^{14}$ Son traité sur la construction des vaisseaux sera soumis par l'Académie

${ }^{11}$ Archives de France, Marine $\mathrm{C}^{7} 40$, fo $22 \mathrm{v}$ et 23.

12 L'ingénieur Levasseur. Voir Emile Salone, La colonisation de la Nouvelle-France (Paris), 389.

13 Fragment de lettre de La Galissonière à Duhamel du Monceau, Québec, 28 octobre 1748, American Philosophical Society.

14 Emile Salone, La colonisation de la Nouvelle-France, 388. 


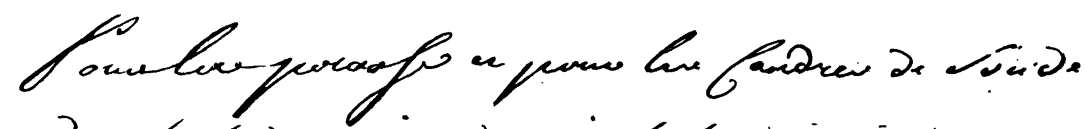

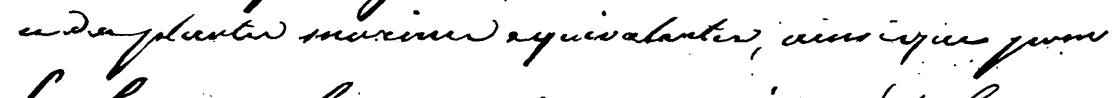

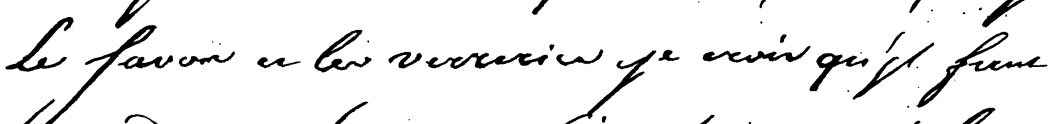

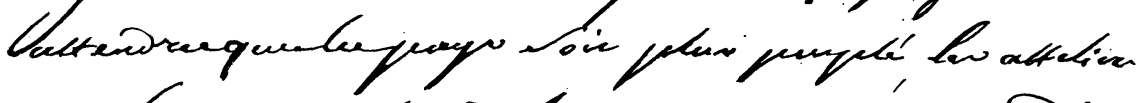

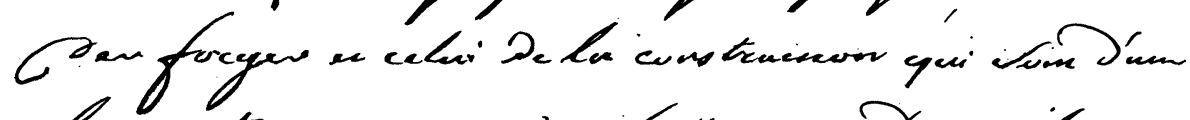

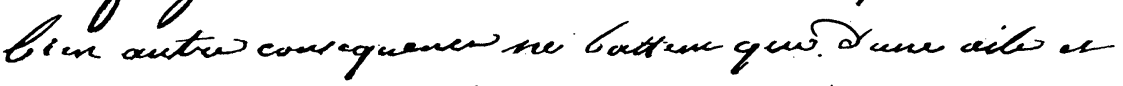

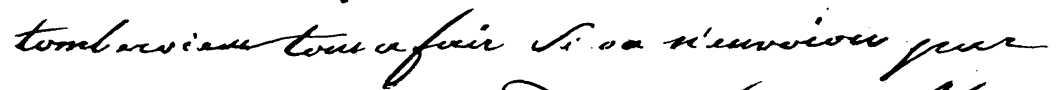

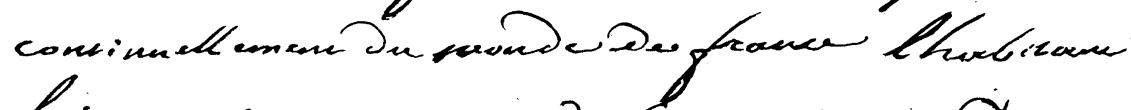

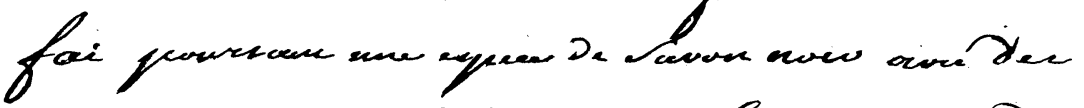

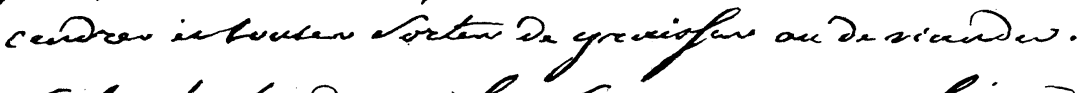

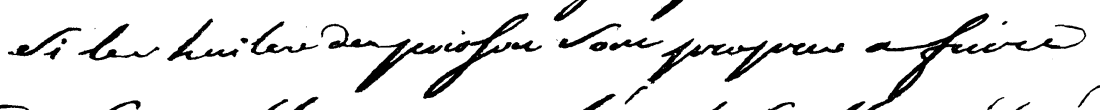

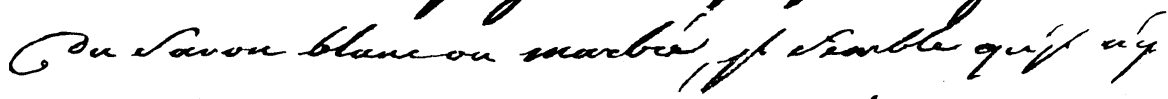

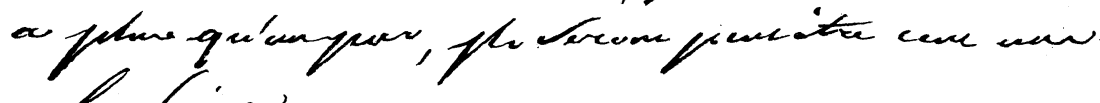
a le facice.

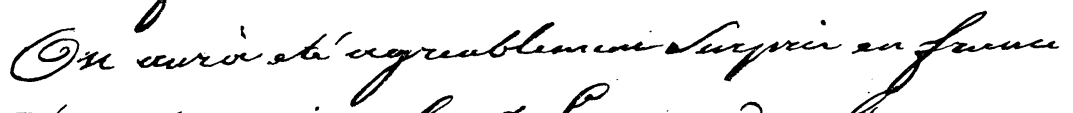

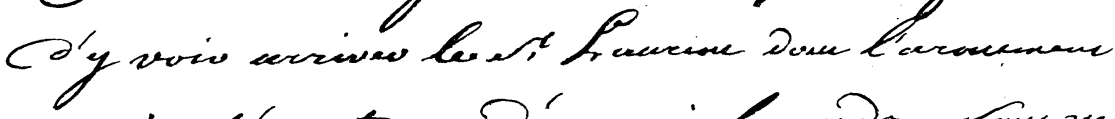

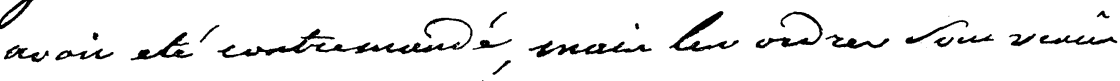

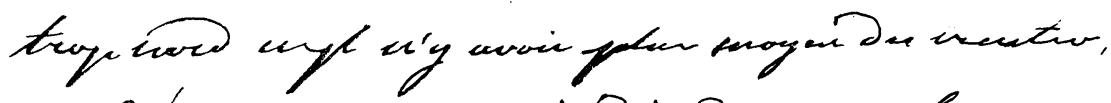

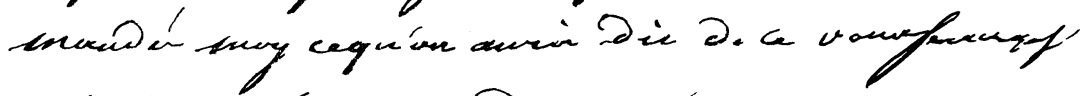

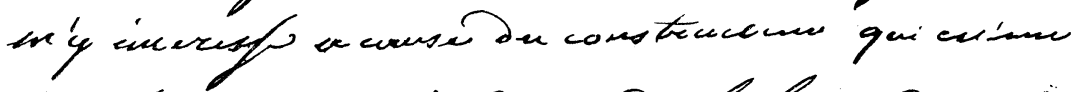

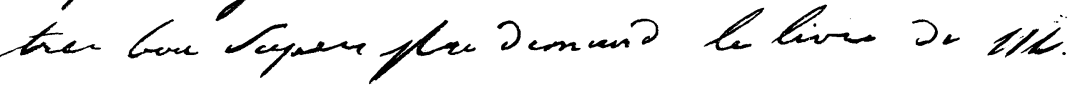

Photocopie d'un extrait de la lettre de La Galissonière à Duhamel du Monceau, Québec, 28 octobre 1748, American Philosophical Society. 
de Marine à l'approbation conjointe de Pierre Bouguer et de Roland-Michel Barrin de La Galissonière qui apposèrent leur signature, en date du 23 septembre $1752 .{ }^{15}$

Les travaux de Bouguer ont un sens général dont il convient de souligner l'importance. Ils mettent à l'épreuve de la méthode expérimentale les formules empiriques de construction navale. L'effort d'amélioration de la technique française portait des fruits, même au Canada. La remarque précitée de La Galissonière sur l'intérêt que suscitait à Québec l'ouvrage de Pierre Bouguer est révélatrice à cet égard.

Université de Montréal

ROLAND LAMONTAGNE

15 Service Historique, Ministère des Armées (Marine), ms. 110. 